\title{
Contrasting chaotic with stochastic dynamics via ordinal transition networks
}

Cite as: Chaos 30, 063101 (2020); https://doi.org/10.1063/1.5142500

Submitted: 13 December 2019. Accepted: 07 May 2020. Published Online: 01 June 2020

F. Olivares (D), M. Zanin (D), L. Zunino (D), and D. G. Pérez (iD)
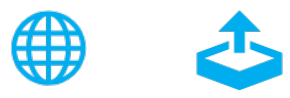

Export Citation

\section{ARTICLES YOU MAY BE INTERESTED IN}

Uncovering episodic influence of oceans on extreme drought events in Northeast Brazil by ordinal partition network approaches

Chaos: An Interdisciplinary Journal of Nonlinear Science 30, 053104 (2020); https:// doi.org/10.1063/5.0004348

\section{Entropy production and fluctuation theorems on complex networks}

Chaos: An Interdisciplinary Journal of Nonlinear Science 30, 053125 (2020); https: // doi.org/10.1063/1.5143031

Asymptotic estimates of SARS-CoV-2 infection counts and their sensitivity to stochastic perturbation

Chaos: An Interdisciplinary Journal of Nonlinear Science 30, 051107 (2020); https:// doi.org/10.1063/5.0008834 


\title{
Contrasting chaotic with stochastic dynamics via ordinal transition networks
}

Cite as: Chaos 30, 063101 (2020); doi: 10.1063/1.5142500

Submitted: 13 December 2019 . Accepted: 7 May 2020.

Published Online: 1 June 2020

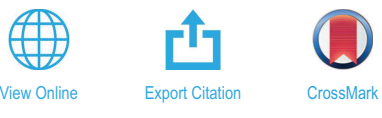

F. Olivares, ${ }^{1, a)}$ (D) M. Zanin, ${ }^{2}$ (D) L. Zunino, ${ }^{3,4}$ (D) and D. G. Pérez ${ }^{1}$

\author{
AFFILIATIONS \\ ${ }^{1}$ Instituto de Física, Pontificia Universidad Católica de Valparaiso (PUCV), 23-40025 Valparaíso, Chile \\ ${ }^{2}$ Centro de Tecnología Biomédica, Universidad Politécnica de Madrid, Campus de Montegancedo, Pozuelo de Alarcón, 28223 \\ Madrid, Spain \\ ${ }^{3}$ Centro de Investigaciones Ópticas (CONICET La Plata-CIC), C.C. 3, 1897 Gonnet, Argentina \\ ${ }^{4}$ Departamento de Ciencias Básicas, Facultad de Ingeniería, Universidad Nacional de La Plata (UNLP), 1900 La Plata, Argentina
}

a) Author to whom correspondence should be addressed: felipe.olivares@pucv.cl

\begin{abstract}
We introduce a representation space to contrast chaotic with stochastic dynamics. Following the complex network representation of a time series through ordinal pattern transitions, we propose to assign each system a position in a two-dimensional plane defined by the permutation entropy of the network (global network quantifier) and the minimum value of the permutation entropy of the nodes (local network quantifier). The numerical analysis of representative chaotic maps and stochastic systems shows that the proposed approach is able to distinguish linear from non-linear dynamical systems by different planar locations. Additionally, we show that this characterization is robust when observational noise is considered. Experimental applications allow us to validate the numerical findings and to conclude that this approach is useful in practical contexts.
\end{abstract}

Published under license by AIP Publishing. https://doi.org/10.1063/1.5142500

Ordinal patterns, i.e., the permutations that have to be applied to segments of a time series to sort their values, have been shown to be an invaluable instrument to analyze the nature of dynamical systems, both numerical and experimental. Some limitations are nevertheless inherent. Specifically, the amount of information captured by each segment is constrained by its length; yet, working with longer segments requires the availability of even longer time series, in order to reach statistically significant results. Instead of considering longer segments, a natural solution involves the analysis of how the ordinal patterns of consecutive (but not overlapping) segments are related, i.e., the analysis of the transitions between these ordinal patterns. We here leverage on this concept to propose a novel plane representation, describing the global and local predictability of these ordinal transitions. We show how the location of a time series in this plane is related to the properties of its dynamics, e.g., non-linearities and self-correlations, and can thus be used to discriminate different dynamical systems. The sensitivity of this method is such that it is able to correctly characterize chaotic maps that are indistinguishable from stochastic processes by using other approaches based on ordinal patterns. We further apply this methodology to real-world time series, representing natural (sunspots dynamics), technical (lasers), and economical (stock markets) systems.

\section{INTRODUCTION}

Time series analysis and characterization is relevant to a broad range of research domains, as indicated by the variety of time series studied in different areas of science. One of the main challenges is to establish whether the underlying dynamical process that generates the time series is either deterministic or stochastic. Several methodologies have been proposed for such a purpose. Particularly, the analysis of time series by recourse to information theory tools together with ordinal patterns $s^{1,2}$ have been shown to be a powerful approach. ${ }^{3-6}$ With the same goal in mind, recent works have focused on time series characterization through complex networks, such as (i) proximity networks, ${ }^{7}$ based on mutual proximity of different segments of the time series, (ii) visibility and horizontal visibility graphs, 8,9 and (iii) transition networks. The latter approach is based on mapping a time series to a Markov chain by defining nodes as some motifs determined by a symbolization technique. Over the 
many schemes proposed to symbolize a time series, ordinal patterns stand out because of their simplicity, robustness in noisy environments, and, most importantly, their sensitivity to time causality. While ordinal transition networks have initially been proposed by Small, ${ }^{10}$ the application of ordinal transition probabilities to time series analysis without a direct appeal to the concept of networks is even earlier. ${ }^{11}$

The pioneering work of Small ${ }^{10}$ proposes the discrimination between chaotic maps, flows and stochastic systems by identifying the ordinal pattern length that maximizes the amount of information in the network; this approach, however, lacks a quantitative criterion for distinguishing the dynamics. Recently, a lot of effort has been directed to the analysis of continuous dynamical systems. To name a few, Sun et al. ${ }^{12}$ have combined ordinal transitions with symbols obtained from different amplitude levels for characterizing dynamical transitions; McCullough et al. ${ }^{13}$ introduced a time-lagged generalization of an ordinal network in order to characterize periodic and chaotic dynamics; and Zhang et al. ${ }^{14}$ have introduced an ordinal partition transition network for multivariate time series.

The aim of the present study is to come back to the basic by pursuing the distinction between deterministic and stochastic dynamics via an entropic quantification of the ordinal transitions. This is motivated by the fact that even when ordinal patterns have been shown to be able to distinguish the time series nature for a large list of chaotic maps and representative stochastic systems, there are still some misclassifications. ${ }^{15}$ Following this line, Borges and co-workers ${ }^{16}$ introduced a method based on the probability of selftransitions or loops. They propose to fit the loop probability as a function of the time lag with a non-linear model. If the curve does not fit well, the data are classified as chaotic. Here, we propose to use what we believe is one of the most simplest approaches for characterizing a time series dynamics: the concept of entropy. We introduced a two-dimensional representation space defined by the permutation entropy of the network and the minimum value of the permutation entropy of the nodes, in order to combine global and local dynamical information. By resorting to a list of representative chaotic maps and stochastic systems, we accomplish a distinction between different dynamical behaviors by assigning a position in this informational plane to each system under study. Several practical applications have also been added to illustrate the goodness of this ordinal network tool.

\section{ORDINAL NETWORKS FROM A TIME SERIES}

\section{A. Ordinal patterns}

Given a one-dimensional time series, $\mathcal{X}=\left\{x_{t} ; t=1, \ldots, M\right\}$, the extraction of ordinal patterns first requires the definition of two parameters: the order of the permutation symbols $D \geqslant 2(D \in$ $\mathbb{N}$, the pattern length) and the lag $\tau(\tau \in \mathbb{N}$, the time separation between the values). Next, the time series can be mapped into subsets of length $D$ of consecutive $(\tau=1)$ or non-consecutive $(\tau>1)$ values, generated by $(t) \equiv\left(x_{t}, x_{t+\tau}, \ldots, x_{t+(D-2) \tau}, x_{t+(D-1) \tau}\right)$, which assigns to each time $t$ the $D$-dimensional vector of values at times $t$, $t+\tau, \ldots, t+(D-1) \tau$. Clearly, more temporal information is incorporated into the vectors as $D$ increases. Subsequently, each element of the vector from zero to $D-1$ is replaced by a number related to its relative ranking-i.e., the smallest value by one and the largest one by $D$. Equal values in the time series are usually ranked according to their temporal order. This is justified if the values of $\mathcal{X}$ have a continuous distribution so that equal values are very unusual. ${ }^{17}$ Taking into account that there are $D$ ! possible permutations for a $D$-dimensional vector, the condition $M \gg D$ ! must be satisfied in order to obtain a reliable statistics. ${ }^{1}$ Bandt and Pompe suggest in their cornerstone paper to work with $3 \leq D \leq 7$ and $\tau=1$. Nevertheless, it has been demonstrated that the analysis with lagged data points, i.e., $\tau \geq 2$, can be useful for reaching a better comprehension of the underlying dynamics. ${ }^{4,18,19}$ Essentially, by changing the value of the lag $\tau$, different time scales are being considered, as this parameter physically corresponds to multiples of the sampling time of the signal under analysis.

All ordinal patterns can eventually appear in a time series generated by a stochastic process with $M \rightarrow \infty$. Particularly, when dealing with correlated stochastic processes, some ordinal patterns may not appear due only to statistical limitations. Temporal correlations in the data make some ordinal patterns more probable than others; hence, longer datasets are needed to observe all the ordinal patterns. ${ }^{20}$ In this context, unobserved ordinal patterns in a time series caused by finite size effects are usually referred as missing. ${ }^{20}$ In the case of time series generated by deterministic dynamics, not all possible ordinal patterns can effectively be materialized into orbits, which in a sense makes them forbidden. This fact was analytically demonstrated for deterministic one-dimensional maps. ${ }^{21-23}$ The existence of forbidden patterns has also been numerically concluded for a large representative set of chaotic maps ${ }^{15}$ and for discrete sampled continuous chaotic dynamics. ${ }^{24-26}$

\section{B. Ordinal transitions}

By counting the number of times each ordinal pattern $\pi_{i}$ appears in the symbolic series divided by the total number of ordinal patterns $L$, we can compute a probability distribution function

$$
p_{i}=\frac{\#\left(\pi_{i}\right)}{L}, \quad i \in[1, D !],
$$

where $\#\left(\pi_{i}\right)$ stands for the cardinality of $\pi_{i}$. For a time series of length $M$, the number of patterns generated is $L=M-(D-1) \tau$. The transition probability between two non-overlapped ordinal patterns, $p_{i \rightarrow j}$, where $i$ and $j$ can take any value of the set $\{1, \ldots, D !\}$, is defined as follows:

$$
p_{i \rightarrow j}=\frac{\#\left(\pi_{l}=i, \pi_{l+1}=j\right)}{\#\left(\pi_{l}=i\right)}, \quad l \in[1, L-D \times \tau] .
$$

Here, we consider self-transitions. All transition ordinal probabilities are expected to be equal to $1 / D$ !, corresponding to a Markov process between pattern pairs, ${ }^{11}$ for a totally uncorrelated time series with $M \rightarrow \infty$. The condition $L \gg D ! \times D !$ must hold in order to have good statistics. The probability matrix of Eq. (2) could be thought as a weighted adjacent matrix of the network. Figure 1(a) illustrates how to create non-overlapped ordinal transitions from a simple example for $D=3$ and $\tau=1$. A graphical representation of the six possible patterns is shown in Fig. 1(b), following the convention of Parlitz et al. ${ }^{27}$ to list the ordinal patterns. Figure 1(c) shows the ordinal transition probability matrix for the example data sequence depicted in Fig. 1(a). Edges assigned between the nodes 
(a)

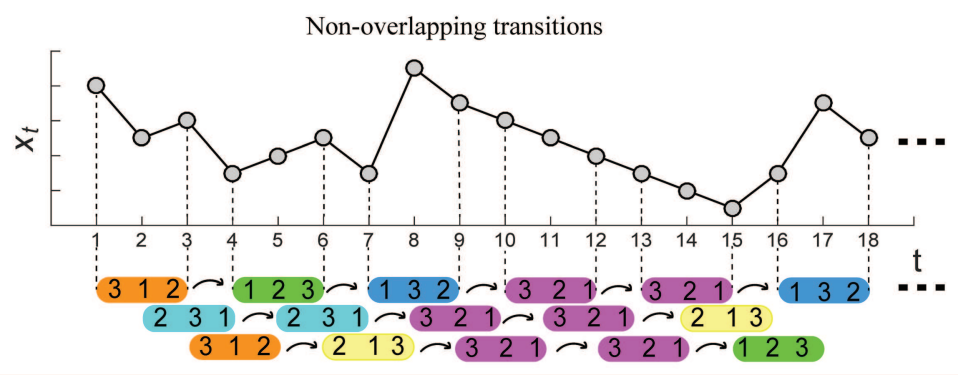

(c)

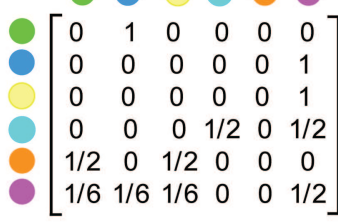

(d)

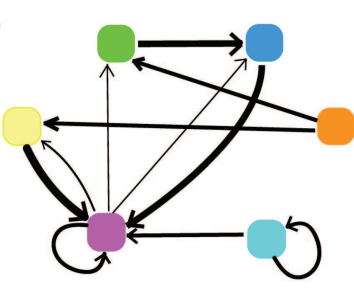

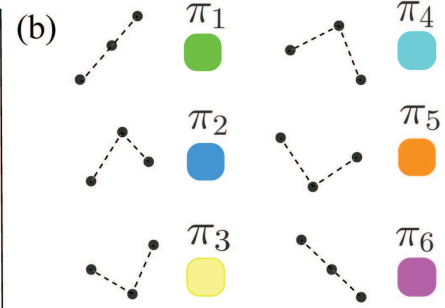

(e) Overlapping ordinal patterns

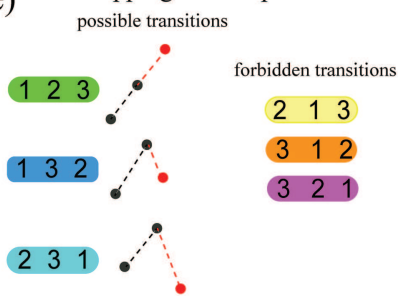

FIG. 1. An example to illustrate the methodology of mapping a time series into a ordinal network. (a) Non-overlapping ordinal transition construction from a time series using $D=3$ and $\tau=1$, (b) graphical representation of ordinal patterns for $D=3$, (c) ordinal transition probability matrix $p_{i \rightarrow j}$, and (d) ordinal network constructed from the example in (a). (e) Forbidden and possible ordinal transitions if overlapping transitions are considered.

based on the temporal succession of the non-overlapped ordinal patterns are depicted in Fig. 1(d).

Naturally, the observed ordinal transitions are directly conditioned by the existence of forbidden patterns. Consequently, if $\mathcal{N}_{\mathrm{FP}}$ represents the number of forbidden ordinal patterns for a deterministic time series, the number of forbidden ordinal transitions will be at least $\left(2 D ! \times \mathcal{N}_{\mathrm{FP}}\right)-\mathcal{N}_{\mathrm{FP}}^{2}$. This value gives a lower bound because in addition to those unobserved ordinal transitions directly coming from forbidden ordinal patterns, there could be more unrealizable ordinal transitions due to either determinism or finite size effect. As an illustrative example, we focus on the chaotic dynamics of the logistic map for $r=4$. Ordinal transition probabilities for $D=3$, $\tau=1$, and $M=10^{7}$ are depicted in Fig. 2. It is known from theory that this chaotic dynamics has one forbidden ordinal pattern $\left(\pi_{6}\right)$ when using $D=3,{ }^{22}$ which implies $(2 * 3 ! \times 1)-1^{2}=11$ forbidden ordinal transitions (highlighted with red dots). However, a numerical simulation suggests a total of 16 forbidden ordinal transitions and, thus, that five additional ones are due to determinism (highlighted with white dots). Furthermore, it is expected to find chaotic maps for which forbidden ordinal transitions are observed for a given value of $D$ even though no forbidden ordinal patterns exist for that pattern length. For instance, this is the case of the linear congruential map for $D=5$, which will studied in detail in Sec. III.

It is important to point out that, when considering overlapping to build the ordinal transition between ordinal patterns from a data sequence, there exist forbidden transitions coming from the way in which the ordinal transitions are constructed. Figure 1(e) illustrates this fact for $D=3$ and $\tau=1$. Considering the example data sequence, the possible ordinal patterns which can follow the first one $\{312\}$ are determined by the overlap. Thus, only three ordinal patterns can materialize: $\{123\},\{132\}$, or $\{231\}$, leaving the rest of possible permutations as forbidden. Due to this fact, any dynamics have forbidden ordinal transitions when an overlapping scheme is implemented. Actually, totally uncorrelated dynamics are characterized by non-equiprobable ordinal transition probabilities. We have considered a non-overlapping recipe in order to avoid this drawback.

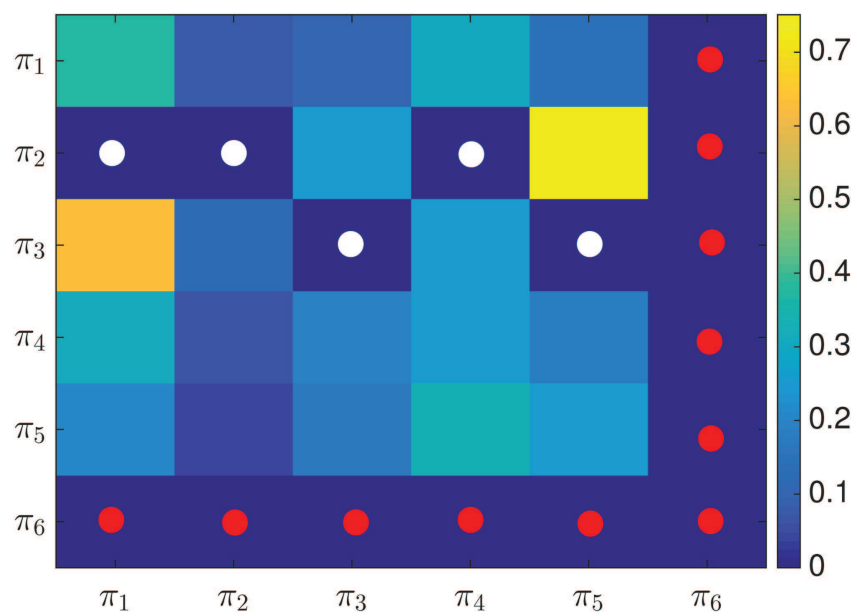

FIG. 2. Ordinal transition probabilities for the logistic map with a control parameter $r=4$, which gives a totally chaotic dynamics, for $D=3, \tau=1$, and $M=10^{7}$. Red dots indicate null probability ordinal transitions coming from forbidden ordinal patterns while white dots point out the "new" forbidden ordinal transitions. 


\section{Ordinal transition entropy}

When considering a probability distribution function (PDF), this can be characterized by resorting to the classical Shannon entropy. Particularly, the Shannon entropy evaluated on the ordinal pattern PDF, given by Eq. (1), corresponds to the so-called permutation entropy. ${ }^{1}$ Similarly, the permutation entropy of the node $i$ is defined in its normalized form as

$$
\mathcal{H}_{i}=-\frac{1}{\ln D !} \sum_{j=1}^{D !} p_{i \rightarrow j} \ln p_{i \rightarrow j}, \quad i \in[1, D !]
$$

which follows the concept of node entropy as defined by West et al. ${ }^{28}$ - note that self-transitions are being considered here. Each entropy value $\mathcal{H}_{i}$ quantifies the randomness of locals transitions. The conditional permutation entropy ${ }^{29}$ is defined as

$$
\mathcal{H}_{n}=\sum_{i=1}^{D !} p_{i} \mathcal{H}_{i}
$$

which is the expected value of the entropy of the node $i$ as averaged over the stationary distribution of the ordinal network, $p_{i}$ [Eq. (1)]. ${ }^{30}$ Hereafter, we will refer to the conditional permutation entropy as the permutation entropy of the network, since it characterizes the ordinal network from a global perspective. For more details about different metric definitions of an ordinal network and how they relate to each other, please see Ref. 30.

In this work, we introduce a representation space by referring to local vs global ordinal transition entropic quantifiers. Its horizontal and vertical axes are the permutation entropy of the network and the minimum permutation entropy of the nodes, respectively. If the system under analysis lies in a very ordered state, being thus characterized by few ordinal patterns and, consequently, few ordinal transitions, its associated position in the plane will be at the bottom, near the left corner, since it will have forbidden ordinal transitions producing several null values of the permutation entropy of the nodes $\left(\min \left(\mathcal{H}_{i}\right)=0\right)$, together with low value of the permutation entropy of the network $\left(\mathcal{H}_{n} \rightarrow 0\right)$. For deterministic systems, one expects that, if they have forbidden ordinal patterns for the selected pattern length, they locate at the bottom of the plane but showing medium-to-high values of the network permutation entropy. On the other hand, for a very disordered system, the entropic metrics will be located near to the top right corner, since all ordinal transitions are expected to be almost equiprobable. To get insight into this entropic representation plane, we have performed a simulation of $10^{5}$ random transition probability matrices, with $D=2$ and hence four possible transitions, and with elements of the transition matrices drawn from uniform distributions in the range $\left[0,10^{5}\right]$. Note that this is not meant to simulate a random process, which would yield normally distributed values; instead, these matrices represent all possible outcomes of the analysis. Results are shown in Fig. 3, where it can be observed that the diagonal creates an upper bound and all instances span below it.

\section{NUMERICAL APPLICATIONS}

In this section, we focus on the distinction between stochastic and chaotic systems. Concretely, we tackle the following chaotic maps: ${ }^{31}$

(1) Logistic map: $x_{n+1}=r x_{n}\left(1-x_{n}\right)$ in the chaotic regime $r=4$, with initial condition $x_{0}=0.1$ and Lyapunov exponent $\lambda=\ln [2]$

(2) Sine map: $x_{n+1}=A \sin \left(\pi x_{n}\right)(A=1)$, with initial condition $x_{0}=0.1$ and Lyapunov exponent $\lambda \simeq 0.689067$

(3) Cubic map: $x_{n+1}=A x_{n}\left(1-x_{n}^{2}\right)(A=3)$, with initial condition $x_{0}=0.1$ and Lyapunov exponent $\lambda \simeq 1.0986122883$;

(4) Cusp map: $x_{n+1}=1-A \sqrt{\left|x_{n}\right|}(A=2)$, with initial condition $x_{0}=0.5$ and Lyapunov exponent $\lambda=0.5$;

(5) Gauss map: $x_{n+1}=1 / x_{n}, \bmod (1)$, with initial condition $x_{0}$ being an uniformly independent identically distributed (iid) random variable $\in(0,1)$ and Lyapunov exponent $\lambda \simeq 2.373445$;

(6) Linear congruential generator: $x_{n+1}=A x_{n}+B \bmod (C)$ $(A=7141, B=54773, C=259200)$, with initial condition $x_{0}=0$ and Lyapunov exponent $\lambda=\ln |A|=8.873608101$;

(7) Sinai map: $x_{n+1}=x_{n}+y_{n}+\delta \cos \left(2 \pi y_{n}\right) \bmod (1), y_{n+1}=x_{n}$ $+2 y_{n} \bmod (1) \quad(\delta=0.1)$, with initial conditions $x_{0}=0.5$, $y_{0}=0.5$, and Lyapunov exponents $\lambda_{1} \simeq 0.95946, \quad \lambda_{2}$ $\simeq-1.07714$

(8) Dissipative standard map: $x_{n+1}=x_{n}+y_{n+1} \bmod (2 \pi), y_{n+1}$ $=b y_{n}+k \sin \left(x_{n}\right) \bmod (2 \pi)(b=0.1, k=8.8)$, with initial conditions $x_{0}=0.1, \quad y_{0}=0.1, \quad$ and Lyapunov exponents $\lambda_{1} \simeq 1.46995, \lambda_{2} \simeq-3.77254$

(9) Arnold cat map: $x_{n+1}=x_{n}+y_{n} \bmod (1), y_{n+1}=x_{n}+k y_{n}$ $\bmod (1)(k=2)$, with initial conditions $x_{0}=0, y_{0}=1 / \sqrt{2}$, and Lyapunov exponents $\lambda= \pm \ln \left[\frac{1}{2}(3+\sqrt{5})\right]$;

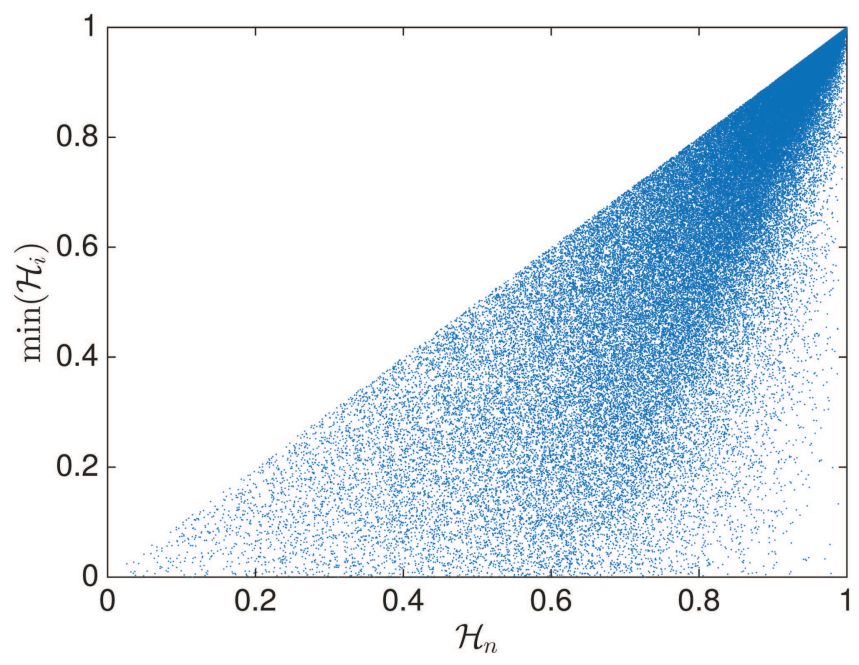

FIG. 3. Locations of $10^{5}$ simulated random transition probability matrices with $D=2$ in the proposed representation space. 
(a)

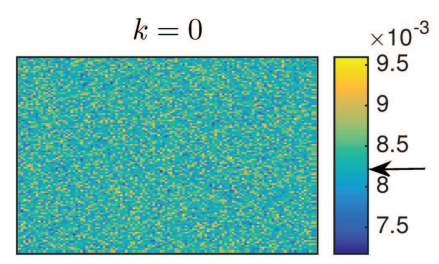

$\mathrm{fBm}, H=0.3$

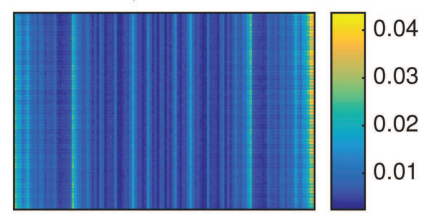

fGn, $H=0.3$

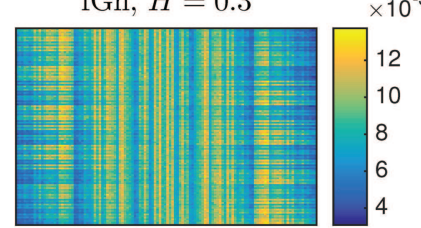

(d)

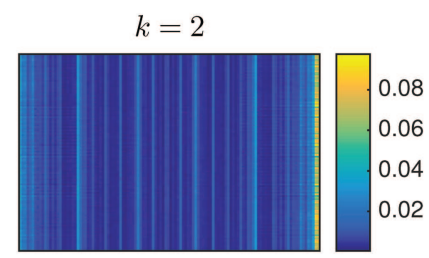

fBm, $H=0.5$

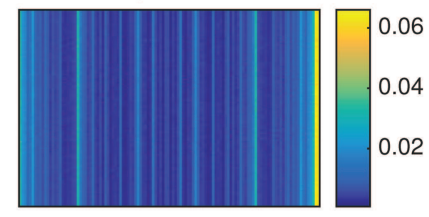

(g)

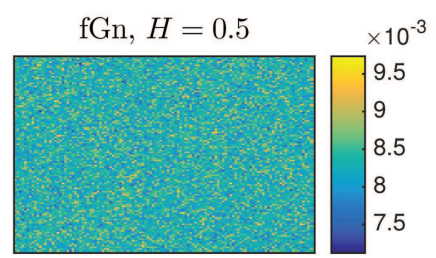

(b)

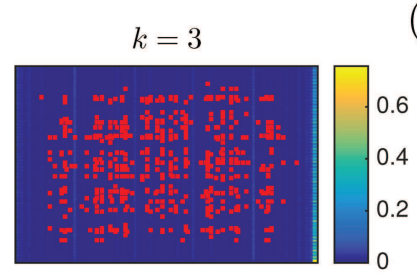

(c)

(e)

fBm, $H=0.8$

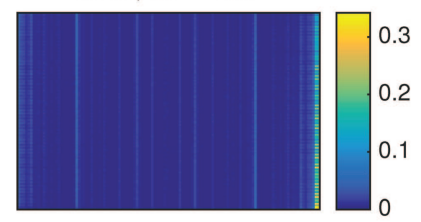

(h)

fGn, $H=0.8$

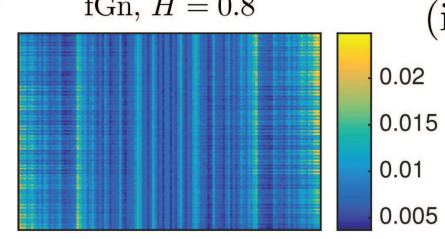

FIG. 4. Ordinal transition probabilities using $D=5$ and $M=10^{7}$ for linear stochastic systems: $k$-noises with (a) $k=0$ (white noise), (b) $k=2$ (Brownian motion), (c) $k=3$ (high temporal correlated noise), fBm with (d) $H=0.3$, (e) $H=0.5$, and (f) $H=0.8$, and fGn with (g) $H=0.3$, (h) $H=0.5$, and (i) $H=0.8$. The equiprobability value $(1 / 5 !=0.0083)$ is depicted with an arrow in (a) as a reference. Red pixels indicate unobserved transitions.

(10) Holmes cubic map: $x_{n+1}=y_{n}, \quad y_{n+1}=-b x_{n}+d y_{n}-y_{n}^{3}$ $(b=0.2, d=2.77)$, with initial conditions $x_{0}=1.6, y_{0}=0$, and Lyapunov exponent $\lambda_{1} \simeq 0.59458, \lambda_{2} \simeq-2.20402$.

It is worth stressing here that some of these chaotic maps have been shown to be particularly problematic to discriminate from stochastic processes by using other approaches based on ordinal patterns [chaotic maps (5) to $(9)^{15}$ ] or based on ordinal networks [chaotic maps $(4)$ and $\left.(10)^{16}\right]$.

Moreover, we have considered a discrete system which presents intermittency: chaotic bursts together with laminar regions: ${ }^{32}$

(11) Schuster maps: $x_{n+1}=x_{n}+x_{n}^{z} \bmod (1)$, with initial conditions: $x_{0}$ iid random variable $\in(0,1)$. As the parameter $z$ decreases, so do the laminar regions. In particular, we used $z=5 / 2,2$, and $3 / 2$.

Additionally, we have considered three kinds of stochastic systems:

(12) Stochastic nonlinear correlated system, ${ }^{33}$ given by

$$
x_{n+1}=a v_{n}+b v_{n-1}\left(1-v_{n}\right),
$$

where $v_{n}$ is a iid random variable $\in(0,1), a=3$, and $b=4$. Also, two linear stochastic systems:

(13) Fractional Brownian motion ( $\mathrm{fBm}$ ) and their increments, fractional Gaussian noise (fGn). ${ }^{35}$ They are characterized by the Hurst exponent $H \in(0,1)$. These processes exhibit temporal correlation for any value of $H$ except for $H=1 / 2$, for which a random walk and a white Gaussian noise are obtained for the former (fBm) and the latter (fGn) model, respectively. For $H>1 / 2$, these processes are long-term correlated, i.e., persistent. On the other hand, when $H<1 / 2$, they present shortterm correlations, i.e., they are antipersistent. It is possible to define a generalized power spectrum of the form $\phi \propto|f|^{-\alpha}$, with $\alpha=2 H+1,1<\alpha<3$ for $\mathrm{fBm}$ and $\alpha=2 H-1,-1<\alpha<1$ for fGn. To simulate the $\mathrm{fBm}$ and fGn time series, we used the function $w f b m$ of MATLAB, which is based on the algorithm proposed by Abry and Sellan. ${ }^{36}$

(14) Noises with $1 / f^{k}$ power spectrum. Starting from a pseudorandom variable with Gaussian distribution probability function, the desired power spectrum is obtained by the Fourier filtering method. Here, the degree of temporal correlations is directly proportional to $k$.

For all the stochastic systems considered, 100 independent realizations of length $10^{7}$ data points were generated with $H \in(0,1)$ with a step 0.05 for the $\mathrm{fBm}$ and their increments and $k \in[0,3]$ with the same step for the $k$-noises. Hereafter, the results reported are the mean over those realizations. For each chaotic map, the same initial conditions and the parameter values detailed by Sprott ${ }^{31}$ were used, and the first $10^{5}$ iterations were discarded to avoid transients behaviors. Considering that we are dealing with discrete systems and neither $\mathrm{fBm}$ nor $\mathrm{fGn}$ have characteristic temporal scales, the time lag is set to $\tau=1$.

Ordinal transition probabilities for the linear stochastic systems, represented by $p_{i \rightarrow j}$, for $D=5$ and $M=10^{7}$ are shown in 


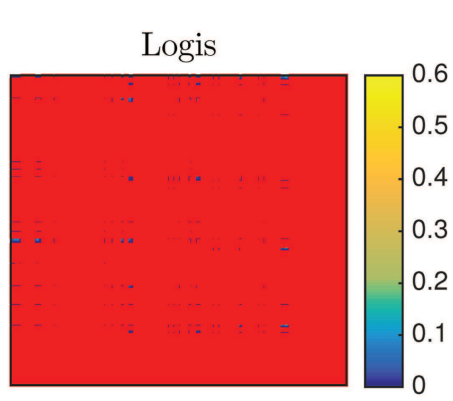

(a)

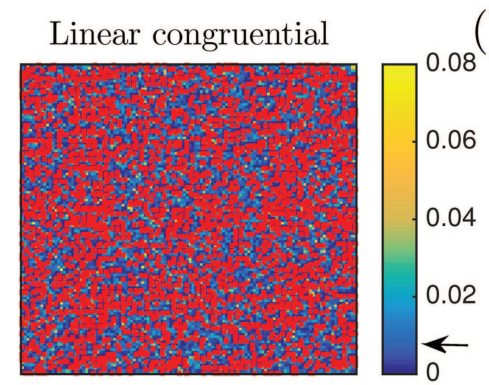

(d)
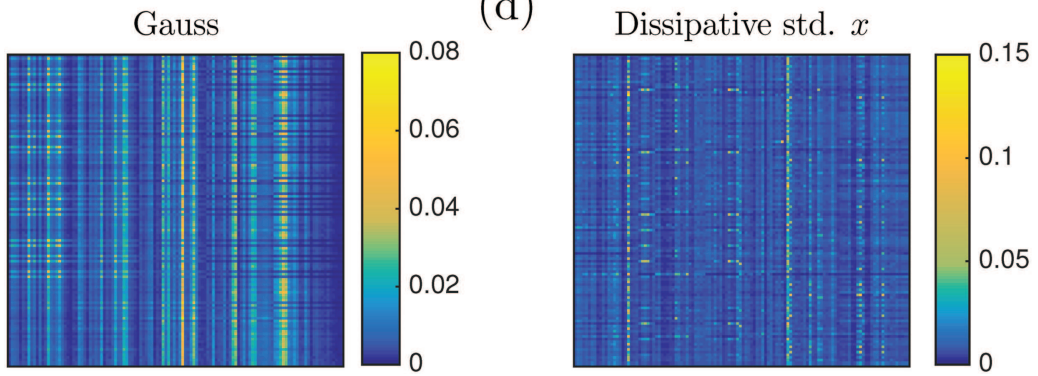

(b)

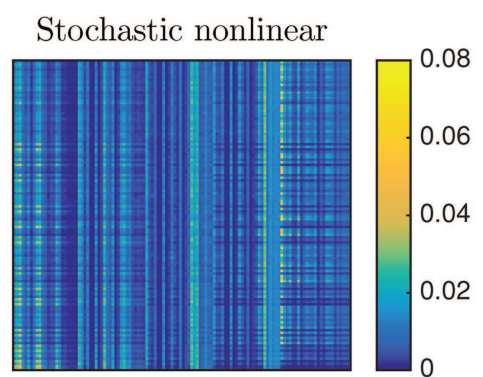

(c)

(e)

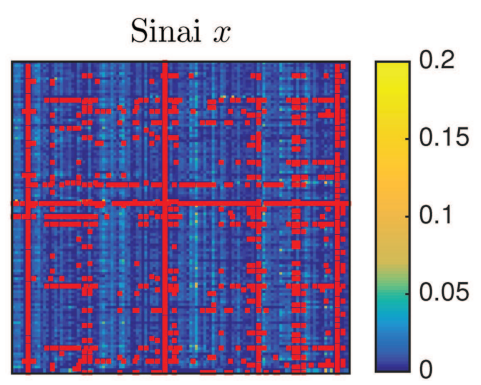

FIG. 5. Ordinal transition probabilities using $D=5$ and $M=10^{7}$ for (a) logistic map, (b) linear congruential map, (c) stochastic nonlinear system, (d) Gauss map, (e) dissipative standard map $x$, and (f) Sinai map $x$. The equiprobability value $(1 / 5 !=0.0083)$ is depicted with an arrow in $(b)$ as a reference. Red pixels indicate unobserved transitions.

Fig. 4. It can be appreciated that for a $k$-noise with $k=0$ and for fGn with $H=1 / 2$ all the ordinal transition probabilities are equiprobable $(1 / D !)$, as expected since both correspond to completely uncorrelated dynamics. On the contrary, in the presence of temporal correlations (long-term $k>0, H>1 / 2$ and short-term $H<1 / 2$ ), privileged ordinal transitions are observed. In particular, higher probabilities are found for the ordinal transitions of all patterns to the all-increasing $(\{12345\})$ and all-decreasing ( $\{54321\})$ ones. Vertical bands are also observed. The regularity observed in the transition matrix for $\mathrm{fBm}$ and their increments comes from the symmetry of the ordinal patterns previously reported by Sinn and $\operatorname{Keller}^{37}$ The $k$-noises seem to share this regularity for $k>0$ (vertical bands). Similar results are observed for $D=3$ and 4 , not shown here. Figure 4(c) shows that there are a considerable number of unobserved transitions (red pixels). Even for long time series, it is normal to find missing ordinal transitions because of the highly persistent dynamics. We have confirmed that this number of unobserved ordinal transitions decreases to zero for longer realizations.

Figure 5 shows the ordinal transition probabilities for some representative chaotic maps and for the nonlinear stochastic system. The logistic map illustrates a case for which most of the ordinal transitions are unobserved due of its forbidden ordinal patterns, $\mathcal{N}_{\mathrm{FP}}=89$ over $5 !=120$ possible ordinal patterns, giving 13439 unobserved ordinal transitions (highlighted in red) coming from the forbidden ordinal patterns. There are only 525 possible ordinal transitions, and some of them have a very high probability of occurrence $(\sim 0.6)$. The number of unobserved ordinal transitions as a function of the data length, for all chaotic maps is listed in Table I. One opposite case, is the Gauss map-see Fig. 5(d) - for which all possible ordinal transitions occur, but at the same time, the matrix $p_{i \rightarrow j}$ shows a clear structure indicating frequent and unlikely ordinal transitions. The same affirmation stands for the dissipative standard map-see Fig. 5(e). For the linear congruential map, its ordinal transition probabilities show a more complex structure, having 2962 unobserved ordinal transitions scattered "randomly" in the matrix-see Fig. 5(b). Surprisingly, this map presents unobserved ordinal transitions while lacking forbidden ordinal patterns, for $5 \leq D \leq 7 .{ }^{15}$ The same affirmation stands for Sinai and Arnold cat maps, with the difference that they present forbidden ordinal patterns for $D \geq 6 .{ }^{15}$ Last, the results for the nonlinear stochastic correlated system are shown in Fig. 5(c). In contrast with the results obtained for linear stochastic processes, although regularities are unobserved, a clear structure emerge. We hypothesize that this is related to the intrinsic nonlinearity. We can conjecture that a transition matrix showing symmetrical structures, as those seen on Fig. 4, comes from a reversible system, as the linear stochastic ones studied here. However, we cannot ensure that any reversible system will produce a transition matrix with symmetrical structures. Similar qualitative structures are found for $D=3$ and 4 , except for the linear congruential map, for which equiprobable ordinal transitions are found for $D=3$. As mentioned before, this map is indistinguishable from an uncorrelated 


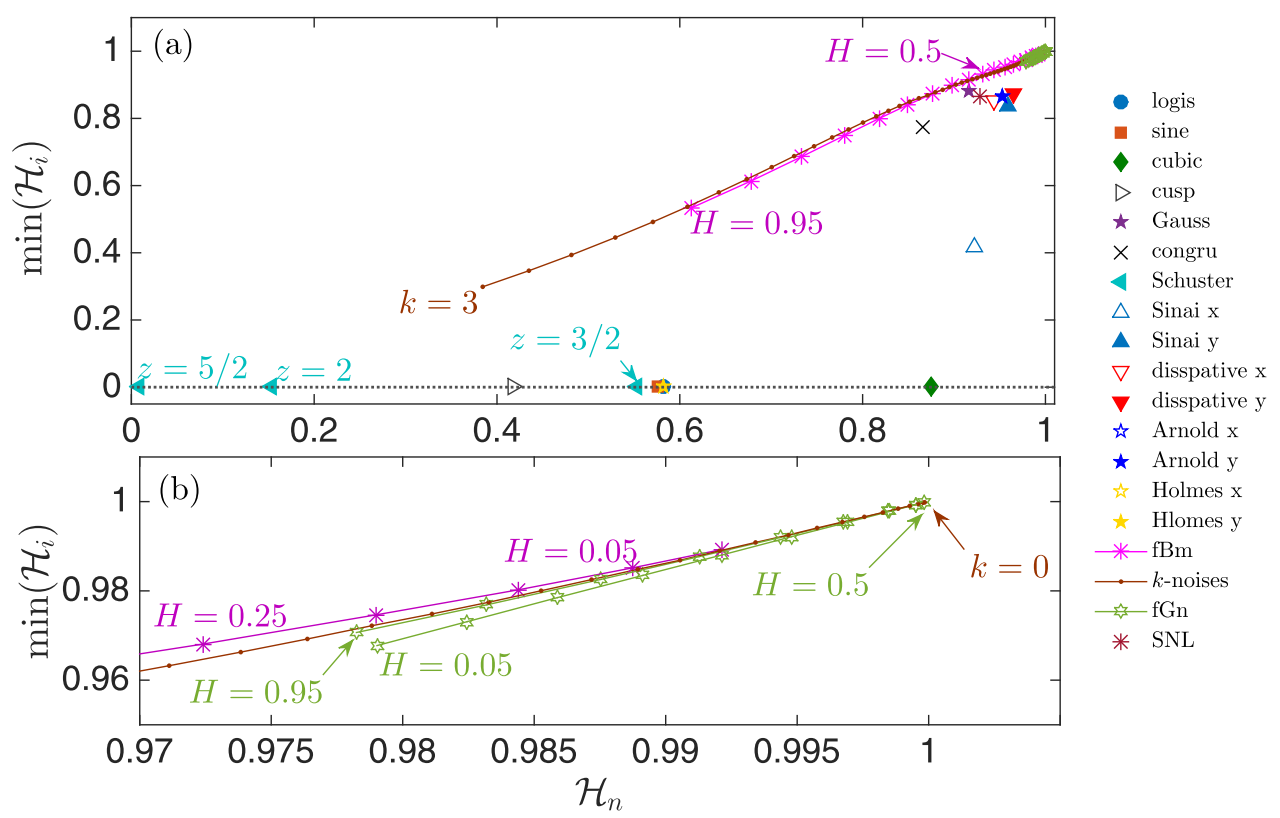

FIG. 6. (a) $\min \left(\mathcal{H}_{i}\right)$ vs $\mathcal{H}_{n}$ using $D=5$ and $\tau=1$ for all the numerical systems studied in this work. (b) Zoom of the upper right corner.

time series by the approach introduced by Rosso et al., ${ }^{15}$ yet ordinal transition probabilities are able to detect its determinism for $D \geq 5$.

With the aim to characterize the ordinal transitions, the permutation entropy of the network, $\mathcal{H}_{n}$ [Eq. (4)], was estimated, to quantify the global disorder in the matrix $p_{i \rightarrow j}$. This is complemented by the minimum permutation entropy per node, $\min \left(\mathcal{H}_{i}\right)$, which accounts for unobserved or very highly probable ordinal transitions. These results are depicted in Fig. 6. All linear stochastic systems locate near the diagonal. For the $k$-noises, as the temporal correlation increases, both quantifiers decrease, as expected. fBm and fGn locations overlap with the one for the $k$-noises. Particularly, times series from fGn exhibit high entropic values-see Fig. 6(b). Both $k$-noise with $k=0$ and fGn with $H=1 / 2$ represent a totally uncorrelated noise and, consequently, both quantifiers are maximal-in agreement with the equiprobable transition probability matrices observed in Figs. 4(a) and 4(h).

Chaotic maps are placed below the linear stochastic systems. Particularly, logistic, sine, cubic, cusp, and Holmes cubic maps locate at the bottom part of the plane with medium/high entropic values. The presence of forbidden ordinal patterns (see last column of Table I) leads to null values of $\min \left(\mathcal{H}_{i}\right)$. On the other hand, when a chaotic map has no forbidden ordinal patterns, forbidden $/$ missing ordinal transitions for a given $D$ can still happen-see Table I. The location obtained for the linear congruential map is the most surprising, since it is not only possible to discriminate it from white noise, but also from correlated stochastic processes, for $D \geq 5$. This is the first time that a discrimination between this "pathological" chaotic map and linear stochastic processes is achieved by a representation space defined through quantifiers using the Bandt and
Pompe methodology. It is worth mentioning that Borges et al. ${ }^{16}$ distinguish this map from stochastic systems by using a non-linear fitting approach of the loop probability with $\tau$. Schuster's maps exhibit null values of $\min \left(\mathcal{H}_{i}\right)$ due to their determinism and laminar regions. As the parameter $z$ decreases, so does the size of the laminar regions; consequently, the entropy of the network increases, approaching a location in the vicinity of the chaotic maps. Finally, it is observed that the nonlinear stochastic map shares location with some of the considered chaotic maps. From this result, we can conclude that this representation space is able to distinguish between linear and non-linear stochastic dynamics. Note that meanwhile the observation of the matrix $p_{i \rightarrow j}$ and its unobserved ordinal transitions (Table I) does not deliver a clear discrimination of the underling dynamics, the representation space does.

In order to study the characterization with this representation plane in a more practical scenario, we analyze two relevant situations when dealing with real-world data: finite size effect and observational noise. First, we study the convergence of the permutation entropy of the network as a function of the time series length. These results are depicted in Fig. 7 for (a) white Gaussian noise, (b) logistic map, and (c) a $\mathrm{fBm}$ with $H=0.8$ (as a representative example of a correlated stochastic dynamics). Clearly, as $D$ increases, a larger data sequence is needed to converge to a stable value of the metric. A similar behavior with the time series length is found for the minimum entropy of the nodes. The convergence of the quantifier seems to be slower for a stochastic dynamics. Up to $D=5$, a data length of $10^{5}$ can be set so that a reliable value of $\mathcal{H}_{n}$ can be estimated.

Finally, to recreate a noisy environment, white Gaussian noise of zero mean was added to the original simulated logistic dynamics with $10^{7}$ data points. Different noise levels (NLs), defined by 
TABLE I. Number of unobserved ordinal transitions for different time series length using $D=5$. The last column shows the number of forbidden ordinal patterns.

\begin{tabular}{|c|c|c|c|c|c|c|}
\hline \multirow[b]{2}{*}{ Map } & \multicolumn{5}{|c|}{ \# unobserved ordinal transitions for $M=$} & \multirow[b]{2}{*}{$\# \mathrm{fp}\left(10^{7}\right)$} \\
\hline & $10^{3}$ & $10^{4}$ & $10^{5}$ & $10^{6}$ & $10^{7}$ & \\
\hline Logistic & 13996 & 13876 & 13875 & 13875 & 13875 & 89 \\
\hline Sine & 14003 & 13881 & 13875 & 13875 & 13875 & 89 \\
\hline Cubic & 13509 & 9967 & 7906 & 7846 & 7846 & 28 \\
\hline Cusp & 14154 & 13915 & 13785 & 13785 & 13785 & 89 \\
\hline Gauss & 13522 & 9211 & 2364 & 59 & 0 & 0 \\
\hline Linear congruential generator & 13446 & 8517 & 3687 & 2962 & 2962 & 0 \\
\hline Sinai $x$ & 13493 & 8863 & 3163 & 1430 & 1131 & 0 \\
\hline Sinai $y$ & 13444 & 8069 & 1074 & 171 & 106 & 0 \\
\hline Dissipative standard $x$ & 13482 & 8511 & 675 & 0 & 0 & 0 \\
\hline Dissipative standard $y$ & 13455 & 8073 & 198 & 0 & 0 & 0 \\
\hline Arnold cat $x$ & 13470 & 8246 & 1637 & 523 & 411 & 0 \\
\hline Arnold cat $y$ & 13465 & 8219 & 1589 & 522 & 412 & 0 \\
\hline Holmes cubic $x$ & 13815 & 12618 & 11600 & 11200 & 11094 & 22 \\
\hline Holmes cubic $y$ & 13815 & 12618 & 11600 & 11200 & 11094 & 22 \\
\hline Schuster $z=5 / 2$ & 14399 & 14181 & 14235 & 13663 & 13219 & 72 \\
\hline Schuster $z=2$ & 14399 & 13963 & 13403 & 13052 & 13011 & 72 \\
\hline Schuster $z=3 / 2$ & 13930 & 13248 & 13026 & 13010 & 13010 & 72 \\
\hline Stochastic non-linear correlated system & 14400 & 14400 & 14400 & 251 & 0 & 0 \\
\hline
\end{tabular}
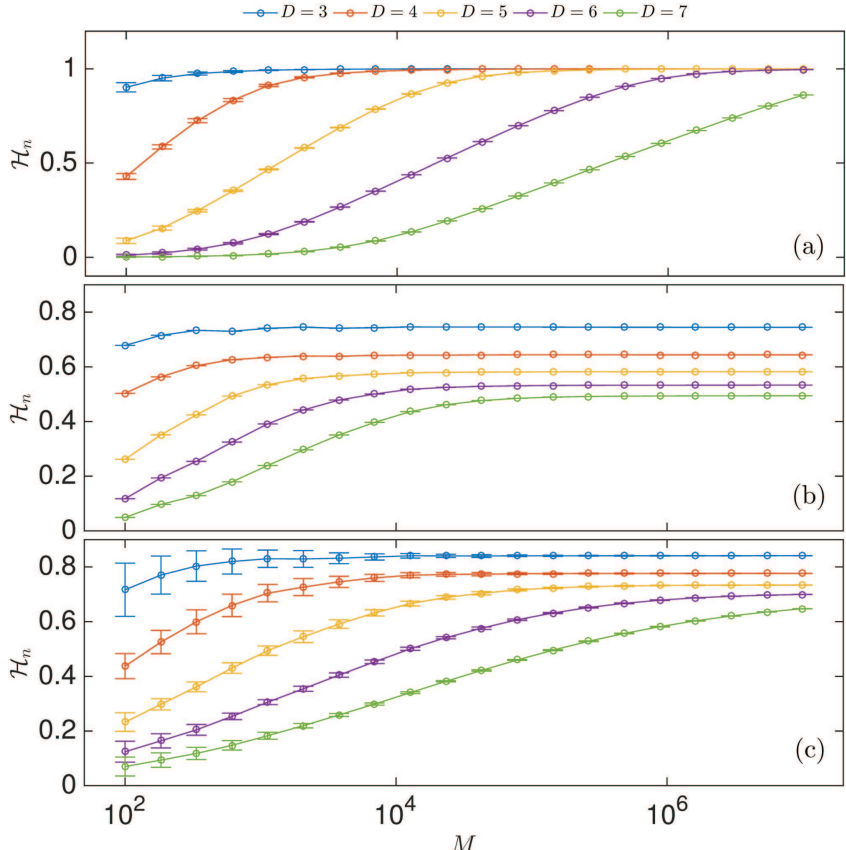

FIG. 7. Evolution of $\mathcal{H}_{n}$ as a function of the time series $M$ with $D=3,4,5,6$, and 7 , for (a) white Gaussian noise, (b) logistic map, and (c) fBm with $H=0.8$. Mean and standard deviation (showed as error bars) over 100 independent realizations are depicted. the ratio between the standard deviation of the noise and the original data, were considered. These results are depicted in Fig. 8. As the noise level increases, the position of the noisy logistic map moves from the bottom part of the plane to the linear stochastic line. Only for very high noise levels $(\mathrm{NL} \sim 1)$ the localization overlaps with that obtained for the linear stochastic systems. Even when $\mathrm{NL}=1$, the noisy chaotic dynamic can be distinguished from a totally uncorrelated dynamic-see the inset in Fig. 8.

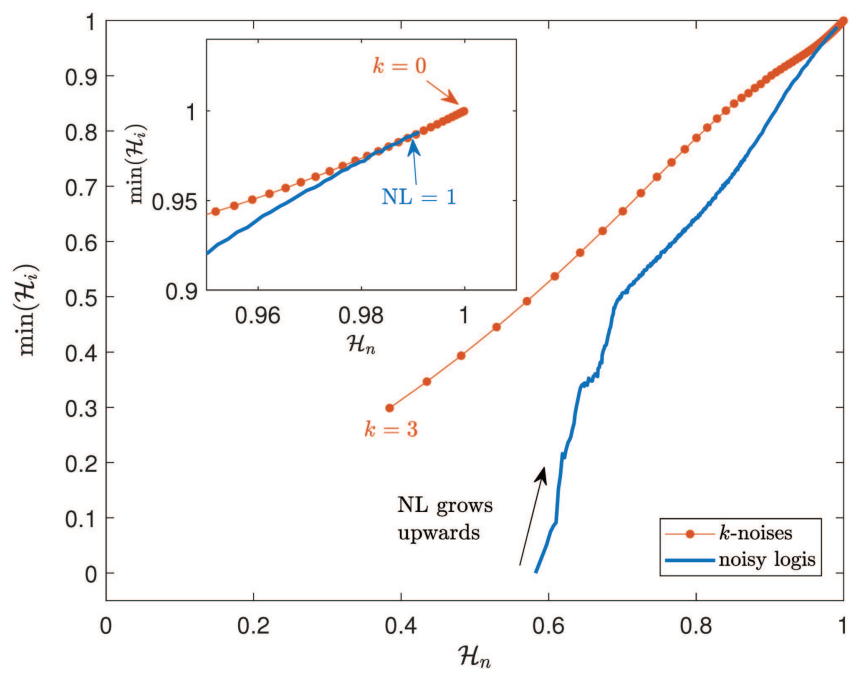

FIG. 8. $\min \left(\mathcal{H}_{i}\right)$ vs $\mathcal{H}_{n}$ using $D=5$ for the noisy logistic dynamics. $k$-noises are included as a reference for linear stochastic systems. The inset corresponds to a zoom of the upper right corner of the plane. 


\section{EXPERIMENTAL APPLICATIONS}

To illustrate the performance of the proposed representation plane with real-world data, we have applied it to four different experimental scenarios.

\section{A. Chaotic laser data}

We have first considered real-world data coming from an opto-electronic oscillator with delay. This experimental system can generate intensity pulsations that are typical for the Ikeda scenario, including a period doubling route to chaos. ${ }^{38,39}$ Here, we focus on the chaotic dynamics of the system. ${ }^{19}$ The data have been acquired with a sampling rate of $f_{s}=500 \mathrm{MSamples} / \mathrm{s}$ and a resolution of 8 bits, for a time long enough to obtain 25 time series of one million data points each. For further details of the experiment, please see Ref. 19. This experimental dataset is a good example of a chaotic dynamic contaminated with observational noise, which is mainly originated from the digitization of the oscilloscope; it is, therefore, perfect to validate our findings on noisy chaotic dynamics.

Since the bandwidth of the experimental system is narrower than the one of the detection apparatus, we benefit from the oversampling to smooth the original time series. ${ }^{19}$ We perform a simple moving average using different window lengths in order to clean the observational noise. These results are shown in Fig. 9. As expected, the original raw measured data locate near the linear stochastic reference due to the observational noise. On the other hand, as the window size of the moving average increases, the location of the quantifiers evolves in a way similar to the one obtained for the noisy logistic dynamics-see Fig. 8. By filtering the observational noise, the proposed representation space is able to capture the determinism of the measured time series, until reaching medium values for

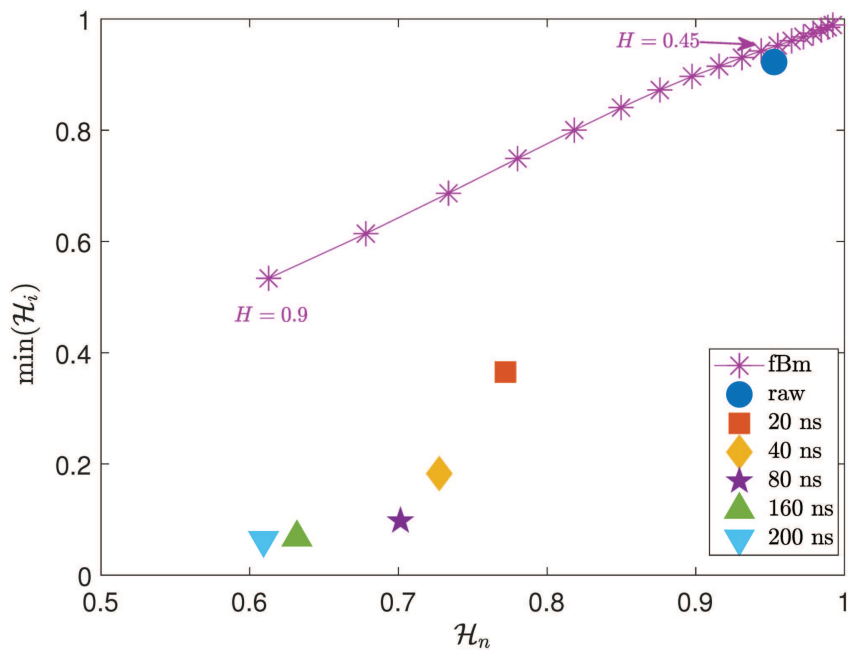

FIG. 9. $\min \left(\mathcal{H}_{i}\right)$ vs $\mathcal{H}_{n}$ using $D=5$ for the chaotic laser data. Average of the quantifiers over 25 independent realizations are depicted. Locations of fBm with the same length $\left(M=10^{6}\right.$ data points) are included as a reference of linear stochastic systems. the network permutation entropy and small values for the minimum permutation entropy of the nodes.

\section{B. Stock markets}

One of the tenets of financial economics is the so-called efficient-market hypothesis, according to which prices in efficient stock markets should follow random walks. ${ }^{40}$ However, deviations from this ideal model, violating either the independence or Gaussian assumptions, have been found in empirical studies. ${ }^{41-44}$ Moreover, it has been shown that stock market indices have a multifractal nature. ${ }^{42}$ Here, we consider the logarithmic price returns of 48 stock market indices of different countries. All data were collected from the Datastream platform. ${ }^{45}$ Daily prices starting on January 3, 2000 and ending on May 27, 2016 are considered (4280 observations). These stock markets are classified as developed (20 stock market indices), emerging (17 stock market indices) or frontier (11 stock market indices) using the Morgan Stanley Capital Index. ${ }^{46}$

Results from the integrated logarithmic price returns are depicted in Fig. 10(a). It can be observed that most of the markets locate below the $\mathrm{fBm}$ reference and, therefore, below the linear stochastic reference. Additionally, we have analyzed 100 independent shuffled realizations of the logarithmic price returns, in order

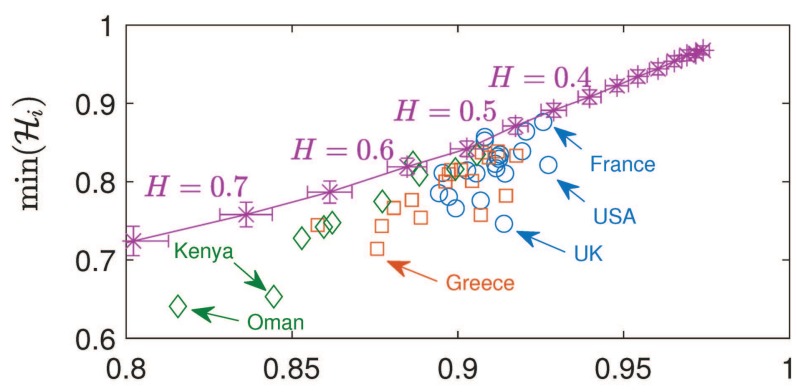

(a)

(b)

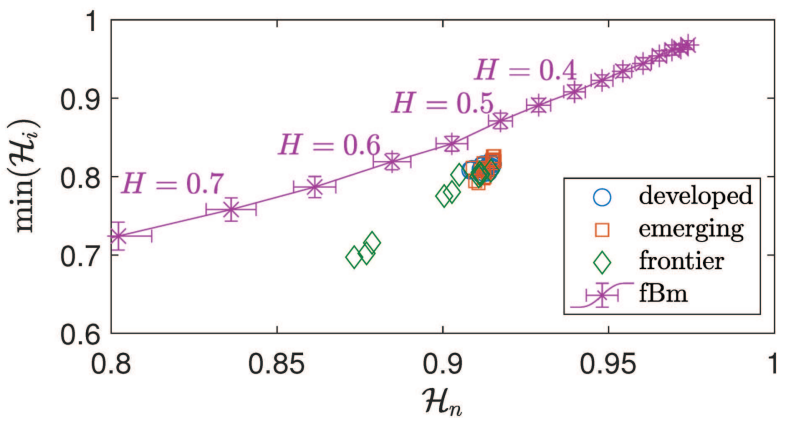

FIG. 10. $\min \left(\mathcal{H}_{i}\right)$ vs $\mathcal{H}_{n}$ for developed (blue circles), emerging (red squares), and frontier (green diamonds) stock markets for $D=4$ for (a) integrated log prices returns and (b) integrated shuffled realizations. Locations of fBm with the same length ( $M=4280$ data points) are included as a reference of linear stochastic processes (mean and one standard deviation, showed as error bars, over 100 independent realizations are shown for each Hurst exponent). 
to destroy all linear and nonlinear correlations but maintaining the same amplitude probability distribution. The analysis of the integrated surrogate is depicted in Fig. 10(b). It is observed that the position of the quantifiers still locates all markets below the linear stochastic reference. We hypothesize that the departure from the $\mathrm{fBm}$ model reflects the presence of a "static non-linearity" due to the probability distribution of the data, ${ }^{47}$ which also is the source of the multifractal nature of the data. These results are in line with those found by implementing a totally different methodology.

\section{Laser beam wandering}

Any laser beam that propagates through the turbulent atmosphere experiments perpendicular displacements to the initial unperturbed direction of propagation. ${ }^{49}$ This phenomenon is commonly known as laser beam wandering because of the dancing that the beam performs over a screen. Particularly, it has been empirically confirmed for isotropic and anisotropic mixtures of hot and cold high-speed air flows that the integrated fluctuations of the laser centroid coordinates can be modeled by a $\mathrm{fBm}$ with a Hurst exponent in the range $1 / 2<H<1$, depending on the velocity of the air flows. ${ }^{50}$ Thus, this seems to be a good example of a measured linear stochastic process.

We here consider an experiment consisting of a laser beam propagating though artificial turbulence. Fluctuations of the centroid position along the vertical and horizontal axes were recorded at $500 \mathrm{~Hz}$ for a fix turbulence intensity. Ten independent measurements of length $M=10^{4}$ data points for eight fans equal speeds were obtained. For further details about the experiments, please see Ref. 50. Results obtained from the integrated measured fluctuations are shown in Fig. 11. It can be observed that both horizontal and

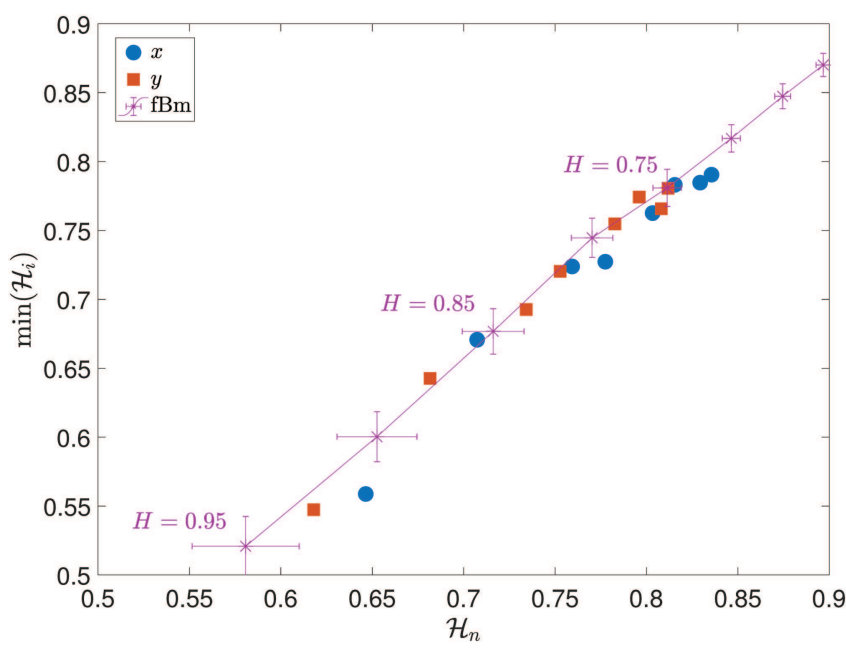

FIG. 11. $\min \left(\mathcal{H}_{i}\right)$ vs $\mathcal{H}_{n}$ for $D=4$. Average over ten independent measurements are depicted for horizontal and vertical coordinates, with each point corresponding to a different flow velocity. Locations of $\mathrm{fBm}$ with the same length $\left(M=10^{4}\right.$ data points) are included as a reference of linear stochastic processes (mean and one standard deviation, showed as error bars, over 100 independent realizations are shown for each Hurst exponent). vertical coordinates overlap with the $\mathrm{fBm}$ process, independently of the air flow velocities. This result permits us to confirm that the fluctuations of the centroid coordinates are compatible with a persistent linear stochastic process.

\section{Sunspot number}

The best known property of the sunspot number time series is its approximate 11 year cycle. However, a lot of effort has been focused on the characterization of the fluctuations mounted on these cycles. ${ }^{51-54}$ Particularly, Hu et al..$^{53}$ have shown a multifractal behavior of the fluctuations with a Hurst exponent equal to 0.74 for short scales.

Here, we analyzed the monthly International Sunspot Number series available at the SIDC's website. ${ }^{55}$ The dataset used in the present analysis spans the January 1749-September 2019 period (3249 data points). Following the work of Zhou and Leung, ${ }^{52}$ we have filtered the monthly sunspot time series by implementing empirical mode decomposition ${ }^{56}$ and focused on the fourth residual signal. ${ }^{52,54}$ Additionally, we have estimated 100 independent surrogate realizations by implementing the iterative amplitude adjusted Fourier transform (IAAFT), in order to produce surrogates with the same amplitude probability distribution and power spectrum, but with potential higher order correlations being randomized. ${ }^{57} \mathrm{We}$ have analyzed the integrated fluctuations for the filtered data and its surrogates. These results are shown in Fig. 12. It is observed that the position of the quantifiers locates the sunspot time series far from the linear stochastic reference $(\mathrm{fBm})$. On the other hand, its surrogates locate very close to a fBm with $H>0.7$. This value of the Hurst exponent is in accordance with those previously reported. ${ }^{51-54}$ From these analyses, we can conclude that the fluctuations of the sunspot

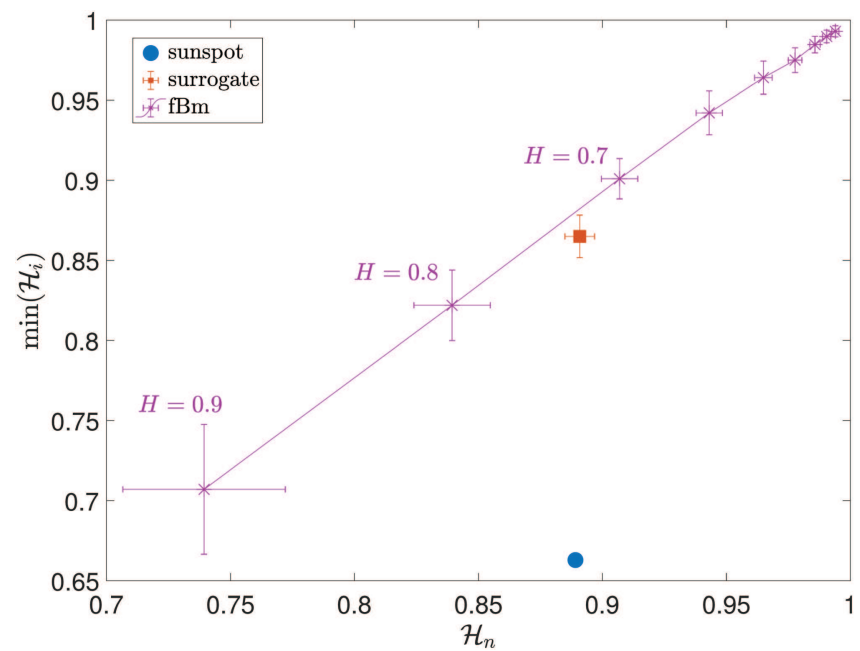

FIG. 12. $\min \left(\mathcal{H}_{i}\right)$ vs $\mathcal{H}_{n}(D=3)$ for the filtered sunspot time series and its surrogate (mean and one standard deviation over 100 independent realizations are depicted). Locations of $\mathrm{fBm}$ with the same length $(M=3249$ data points) are included as a reference of linear stochastic processes (mean and one standard deviation, showed as error bars, over 100 independent realizations are shown for each Hurst exponent). 
time series present non-linear correlations, which is consistent with the observed multifractal nature.

\section{CONCLUSIONS}

By leveraging on the ordinal patterns transitions to construct an ordinal transition network, we have introduced here a two-dimensional representation space, defined by the minimal permutation entropy of the nodes vs the permutation entropy of the network, aimed at capturing local and global features of the dynamics, respectively.

We have contrasted the characterization of chaotic and stochastic dynamical behaviors, represented by finite time series. The numerical analysis of some representative chaotic and stochastic systems supports the claim that a distinction can be made between linear stochastic systems and non-linear deterministic/stochastic dynamics by referring to their planar location in our representation space. Linear stochastic systems considered here overlap near the diagonal and seem to create an upper bound in the plane. On the other hand, chaotic maps and the non-linear stochastic dynamics locate below this linear limit. We also found that this characterization is still observed when chaotic time series are contaminated with observational noise, even for high levels of this contamination. Furthermore, several experimental applications confirm the numerical results and illustrate the applicability of the proposed characterization plane.

\section{ACKNOWLEDGMENTS}

This work was supported by the Comisión Nacional de Investigación Científica y Tecnológica (CONICYT) FONDECYT POSTDOC (Grant No. 3170533). L.Z. gratefully acknowledges financial support from the Consejo Nacional de Investigaciones Científicas y Técnicas (CONICET), Argentina. D.G.P. was supported by the Pontificia Universidad Catolica de Valparaíso (No. PUCV 039.352/19). We thank Dr. Miguel C. Soriano for providing the chaotic laser data.

\section{DATA AVAILABILITY}

Data sharing is not applicable to this article as no new data were created or analyzed in this study.

\section{REFERENCES}

${ }^{1}$ C. Bandt and B. Pompe, "Permutation entropy: A natural complexity measure for time series," Phys. Rev. Lett. 88, 174102 (2002).

${ }^{2}$ M. Zanin, L. Zunino, O. A. Rosso, and D. Papo, "Permutation entropy and its main biomedical and econophysics applications: A review," Entropy 14, 1553-1577 (2012).

${ }^{3}$ O. A. Rosso, H. A. Larrondo, M. T. Martin, A. Plastino, and M. A. Fuentes, "Distinguishing noise from chaos," Phys. Rev. Lett. 99, 154102 (2007).

${ }^{4}$ L. Zunino, M. C. Soriano, and O. A. Rosso, "Distinguishing chaotic and stochastic dynamics from time series by using a multiscale symbolic approach," Phys. Rev. E 86, 046210 (2012).

${ }^{5}$ F. Olivares, A. Plastino, and O. A. Rosso, "Contrasting chaos with noise via local versus global information quantifiers,” Phys. Lett. A 376, 1577-1583 (2012).

${ }^{6} \mathrm{C}$. Kulp and L. Zunino, "Discriminating chaotic and stochastic dynamics through the permutation spectrum test," Chaos 24, 033116 (2014).

${ }^{7}$ J. Zhang and M. Small, "Complex network from pseudoperiodic time series: Topology versus dynamics,” Phys. Rev. Lett. 96, 238701 (2006).
${ }^{8}$ L. Lacasa, B. Luque, F. Ballesteros, J. Luque, and J. C. Nuno, "From time series to complex networks: The visibility graph," Proc. Natl. Acad. Sci. U.S.A. 105, 4972-4975 (2008).

${ }^{9}$ L. Lacasa and R. Toral, "Description of stochastic and chaotic series using visibility graphs,” Phys. Rev. E 82, 036120 (2010).

${ }^{10}$ M. Small, "Complex networks from time series: Capturing dynamics," in 2013 IEEE International Symposium on Circuits and Systems (ISCAS2013) (IEEE, 2013), pp. 2509-2512.

${ }^{11}$ N. Rubido, J. Tiana-Alsina, M. C. Torrent, J. Garcia-Ojalvo, and C. Masoller, "Language organization and temporal correlations in the spiking activity of an excitable laser: Experiments and model comparison," Phys. Rev. E 84, 026202 (2011).

${ }^{12}$ X. Sun, M. Small, Y. Zhao, and X. Xue, "Characterizing system dynamics with a weighted and directed network constructed from time series data," Chaos $\mathbf{2 4}$, 024402 (2014).

${ }^{13}$ M. McCullough, M. Small, T. Stemler, and H. H.-C. Iu, "Time lagged ordinal partition networks for capturing dynamics of continuous dynamical systems," Chaos 25, 053101 (2015).

${ }^{14}$ J. Zhang, J. Zhou, M. Tang, H. Guo, M. Small, and Y. Zou, "Constructing ordinal partition transition networks from multivariate time series," Sci. Rep. 7, 7795 (2017).

${ }^{15}$ O. A. Rosso, F. Olivares, L. Zunino, L. De Micco, A. L. L. Aquino, A. Plastino, and H. A. Larrondo, "Characterization of chaotic maps using the permutation Bandt-Pompe probability distribution,” Eur. Phys. J. B 86, 116 (2013).

${ }^{16}$ J. B. Borges, H. S. Ramos, R. A. F. Mini, O. A. Rosso, A. C. Frery, and A. A. F. Loureiro, "Learning and distinguishing time series dynamics via ordinal patterns transition graphs,” Appl. Math. Comput. 362, 124554 (2019).

${ }^{17}$ D. Cuesta-Frau, M. Varela-Entrecanales, A. Molina-Picó, and B. Vargas, "Patterns with equal values in permutation entropy: Do they really matter for biosignal classification?," Complexity 2018, 1324696 (2018).

${ }^{18}$ L. Zunino, M. C. Soriano, I. Fischer, O. A. Rosso, and C. R. Mirasso, "Permutation-information-theory approach to unveil delay dynamics from timeseries analysis," Phys. Rev. E 82, 046212 (2010).

${ }^{19}$ M. C. Soriano, L. Zunino, L. Larger, I. Fischer, and C. R. Mirasso, "Distinguishing fingerprints of hyperchaotic and stochastic dynamics in optical chaos from a delayed optoelectronic oscillator,” Opt. Lett. 36, 2212-2214 (2011).

${ }^{20}$ L. C. Carpi, P. M. Saco, and O. Rosso, "Missing ordinal patterns in correlated noises,” Phys. A Stat. Mech. Appl. 389, 2020-2029 (2010).

${ }^{21}$ J. M. Amigó, L. Kocarev, and J. Szczepanski, “Order patterns and chaos," Phys. Lett. A 355, 27-31 (2006).

${ }^{22}$ J. M. Amigó, S. Zambrano, and M. A. F. Sanjuán, "True and false forbidden patterns in deterministic and random dynamics," Europhys. Lett. 79, 50001 (2007).

${ }^{23}$ J. M. Amigó, S. Zambrano, and M. A. F. Sanjuán, "Combinatorial detection of determinism in noisy time series," Europhys. Lett. 83, 60005 (2008).

${ }^{24}$ M. McCullough, K. Sakellariou, T. Stemler, and M. Small, "Counting forbidden patterns in irregularly sampled time series. I. the effects of under-sampling, random depletion, and timing jitter," Chaos 26, 123103 (2016).

${ }^{25}$ K. Sakellariou, M. McCullough, T. Stemler, and M. Small, "Counting forbidden patterns in irregularly sampled time series. II. Reliability in the presence of highly irregular sampling," Chaos 26, 123104 (2016).

${ }^{26}$ C. W. Kulp, J. M. Chobot, B. J. Niskala, and C. J. Needhammer, "Using forbidden ordinal patterns to detect determinism in irregularly sampled time series," Chaos 26, 023107 (2016).

${ }^{27}$ U. Parlitz, S. Berg, S. Luther, A. Schirdewan, J. Kurths, and N. Wessel, "Classifying cardiac biosignals using ordinal pattern statistics and symbolic dynamics," Comput. Biol. Med. 42, 319-327 (2012).

${ }^{28}$ J. West, G. Bianconi, S. Severini, and A. E. Teschendorff, "Differential network entropy reveals cancer system hallmarks," Sci. Rep. 2, 802 (2012).

${ }^{29}$ A. M. Unakafov and K. Keller, "Conditional entropy of ordinal patterns," Phys. D Nonlinear Phenom. 269, 94-102 (2014).

${ }^{30}$ M. McCullough, M. Small, H. H. C. Iu, and T. Stemler, "Multiscale ordinal network analysis of human cardiac dynamics," Philos. Trans. R. Soc. A 375, 20160292 (2017).

${ }^{31}$ J. C. Sprott, Chaos and Time-Series Analysis (Oxford University Press, 2003). 
${ }^{32}$ H. G. Schuster and W. Just, Deterministic Chaos: An Introduction (John Wiley \& Sons, 2006)

${ }^{33}$ U. S. Freitas, C. Letellier, and L. A. Aguirre, "Failure in distinguishing colored noise from chaos using the "noise titration" technique," Phys. Rev. E 79, 035201 (2009).

${ }^{34} \mathrm{~J}$. Gao, J. Hu, X. Mao, and W.-W. Tung, "Detecting low-dimensional chaos by the "noise titration" technique: Possible problems and remedies," Chaos Soliton. Fract. 45, 213-223 (2012).

${ }^{35}$ B. B. Mandelbrot and J. W. Van Ness, "Fractional Brownian motions, fractional noises and applications," SIAM Rev. 10, 422-437 (1968).

${ }^{36} \mathrm{P}$. Abry and F. Sellan, "The wavelet-based synthesis for fractional Brownian motion proposed by F. Sellan and Y. Meyer: Remarks and fast implementation," Appl. Comput. Harmon. Anal. 3, 377-383 (1996).

${ }^{37} \mathrm{M}$. Sinn and K. Keller, "Estimation of ordinal pattern probabilities in Gaussian processes with stationary increments," Comput. Stat. Data Anal. 55, 1781-1790 (2011).

${ }^{38}$ L. Larger and J. M. Dudley, "Optoelectron. chaos," Nature 465, 41 (2010).

${ }^{39}$ M. Peil, M. Jacquot, Y. K. Chembo, L. Larger, and T. Erneux, "Routes to chaos and multiple time scale dynamics in broadband bandpass nonlinear delay electrooptic oscillators," Phys. Rev. E 79, 026208 (2009).

${ }^{40}$ B. G. Malkiel, "The efficient market hypothesis and its critics," J. Economic Perspect. 17, 59-82 (2003).

${ }^{41}$ B. B. Mandelbrot, "The variation of certain speculative prices," J. Bus. 36, 394-419 (1963).

${ }^{42}$ L. Zunino, B. M. Tabak, A. Figliola, D. Pérez, M. Garavaglia, and O. Rosso, "A multifractal approach for stock market inefficiency,” Phys. A Stat. Mech. Appl. 387, 6558-6566 (2008).

${ }^{43}$ L. Zunino, B. M. Tabak, D. G. Pérez, M. Garavaglia, and O. A. Rosso, "Inefficiency in Latin-American market indices," Eur. Phys. J. B 60, 111-121 (2007).

${ }^{44}$ M. Zanin, A. Rodríguez-González, E. Menasalvas Ruiz, and D. Papo, "Assessing time series reversibility through permutation patterns," Entropy 20, 665 (2018).
${ }^{45}$ Datastream, see http://financial.thomsonreuters.com/en/products/toolsapplications/trad ing-investment-tools/datastream-macroeconomicanalysis.html. ${ }^{46}$ Morgan Stanley Capital Index, see https://www.msci.com.

${ }^{47} \mathrm{R}$. Rak and D. Grech, "Quantitative approach to multifractality induced by correlations and broad distribution of data," Phys. A Stat. Mech. Appl. 508, 48-66 (2018).

${ }^{48}$ L. Zunino, F. Olivares, A. F. Bariviera, and O. A. Rosso, "A simple and fast representation space for classifying complex time series," Phys. Lett. A 381, 1021-1028 (2017).

${ }^{49}$ L. C. Andrews and R. L. Phillips, Laser Beam Propagation Through Random Media (SPIE Press, Bellingham, WA, 2005), Vol. 152.

${ }^{50}$ G. Funes, F. Olivares, C. G. Weinberger, Y. D. Carrasco, L. Nuñez, and D. G. Pérez, "Synthesis of anisotropic optical turbulence at the laboratory," Opt. Lett. 41, 5696-5699 (2016).

${ }^{51}$ M. S. Movahed, G. Jafari, F. Ghasemi, S. Rahvar, and M. R. R. Tabar, "Multifractal detrended fluctuation analysis of sunspot time series," J. Stat. Mech. Theory Experi. 2006, P02003 (2006).

${ }^{52} \mathrm{Y}$. Zhou and Y. Leung, "Empirical mode decomposition and long-range correlation analysis of sunspot time series," J. Stat. Mech. Theory Experi. 2010, P12006 (2010).

${ }^{53} \mathrm{~J}$. Hu, J. Gao, and X. Wang, "Multifractal analysis of sunspot time series: The effects of the 11-year cycle and Fourier truncation," J. Stat. Mech. Theory Experi. 2009, P02066 (2009).

${ }^{54} \mathrm{D}$. Gulich and L. Zunino, "A criterion for the determination of optimal scaling ranges in DFA and MF-DFA," Phys. A Stat. Mech. Appl. 397, 17-30 (2014).

${ }^{55}$ Sunspot data from the World Data Center SILSO, Royal Observatory of Belgium, Brussels, see http://sidc.oma.be/sunspot-data/.

${ }^{56}$ N. E. Huang, Z. Shen, S. R. Long, M. C. Wu, H. H. Shih, Q. Zheng, N.-C. Yen, C. C. Tung, and H. H. Liu, "The empirical mode decomposition and the Hilbert spectrum for nonlinear and non-stationary time series analysis," Proc. R. Soc. London. Ser. A 454, 903-995 (1998).

${ }^{57}$ T. Schreiber and A. Schmitz, "Improved surrogate data for nonlinearity tests," Phys. Rev. Lett. 77, 635 (1996). 